\title{
O desenvolvimento do e-government na esfera local a partir de dimensões políticas, tecnológicas e sociais
}

\author{
Pedro Antonio Müller \\ Cláudia Tirelli*** \\ Ramon Gil-Garcia***
}

\begin{abstract}
Resumo
Este artigo apresenta um modelo para identificar variáveis com grau de relevância para o desenvolvimento do e-government na esfera regional. Tem como propósitos contribuir para o entendimento do contexto local a partir de informações analíticas e auxiliar o planejamento do e-government por gestores municipais. Também estimula o desenvolvimento regional, auxiliando servidores públicos a entender variáveis relevantes no contexto local, para a resolução de problemas sociais. Para o desenvolvimento do trabalho, foi utilizado o método quantitativo, com a participação de municípios do extremo sul do Brasil. Identificou-se que, na maioria dos municípios, o e-government ainda está em estágios iniciais, e a tecnologia de informação é utilizada basicamente como meio de comunicação. Também constatou-se que as variáveis infraestrutura tecnológica, recursos financeiros, população, orçamento municipal e taxa de analfabetismo têm se mostrado mais propensas a influenciar o estágio de maturidade do e-government.
\end{abstract}

Palavras-chave: E-government. Modelo de maturidade. Tecnologia da informação.

Mestre em Desenvolvimento pela Unijuí e doutor em Desenvolvimento Regional pela Unisc. Professor adjunto do curso de Ciência da Computação na UPF. E-mail: muller@upf.br

** Mestre e doutora em Sociologia pela UFRGS. Professora adjunta do Departamento de Ciências Humanas e do Programa de Pós-Graduação em Desenvolvimento Regional na Unisc. E-mail: ctirelli@ unisc.br

*** Mestre em Administração Pública e Política pelo Centro de Pesquisa e Ensino em Economia no México e $\mathrm{PhD}$ em Administração Pública e Política pela Universidade de Albany. Professor associado do curso de Administração Pública e Política e diretor de pesquisa do Centro de Tecnologia para Governo, Universidade de Albany, SUNY. E-mail: jgil-garcia@ctg.albany.edu

http://dx.doi.org/10.5335/rtee.v24i50.8961

Submissão: 20/04/2018. Aceite: 20/06/2018. 


\section{Introdução}

Vive-se uma época de muitas mudanças, e um dos desafios é o encontro do novo. O processo de transformação da sociedade industrial em sociedade informacional ainda não se completou inteiramente, e está-se cercado por crises e oportunidades. A percepção do presente e a presunção do futuro estão condicionadas a modelos analíticos que sejam tão dinâmicos quanto a realidade em movimento e reconheçam o comportamento sistêmico das variáveis novas que dão uma significação diferente à totalidade (SANTOS, 2015).

As mudanças vêm acompanhadas por complexidades que se apresentam à sociedade e aos gestores públicos como grandes desafios para o desenvolvimento nos aspectos econômicos, políticos e tecnológicos (ROSE; GRANT, 2010). Na esfera pública, o e-government tem sido abordado pela perspectiva tecnológica como forma de instrumentalizar o processo e potencializar as forças locais.

Um dos maiores desafios dos gestores públicos está em fazer com que seus esforços sejam efetivamente reconhecidos pela população. Algumas alternativas para isso são melhorar a qualidade dos serviços (GRIMSLEY; MEEHAN, 2007), envolver cada vez mais os cidadãos nos processos de tomada de decisão e fortalecer a interação entre governo e sociedade (ZOO; LEE; YOON, 2012).

As melhorias no setor público passam por mudanças ou modernizações do próprio Estado no que tange a seus processos. Esse é um tema que tem sido amplamente discutido a partir do new public management, que tem tratado da modernização do Estado de modo que se consigam reduzir a burocracia e produzir mais com menos recursos e em tempo menor (ALFORD; HUGHES, 2008; PINA; TORRES; ROYO, 2008). Diz respeito à busca da eficiência e da eficácia de forma a possibilitar que as ações dos governos se tornem mais efetivas (LUNA-REYES; GIL-GARCIA; ROMERO, 2012; HEEKS, 2002; HOOD, 1995). O foco, portanto, está na mudança dos processos. Nesse contexto, o e-government tem sido colocado como um meio para fazer frente a processos ineficientes de burocratização e como uma ferramenta capaz de suportar e contribuir para as reformas do Estado (LUNA-REYES; GIL-GARCIA; ROMERO, 2012; HEEKS, 2002; HO, 2002) .

$\mathrm{O}$ e-government tem sido utilizado de forma variada por diferentes níveis de governo: internacional, nacional ou federal, estadual, regional ou local (AFRICA, 2012; IRANI; LOVE, 2008; EDMISTON, 2003). A contribuição do e-government ocorre desde o suporte às operações, voltadas principalmente às necessidades do próprio governo, em um nível mais básico, até como instrumento de participação 
democrática por meio do uso de ferramentas interativas (escolha de prioridades de investimentos, etc.) (ALMARABEH; ABUALI, 2010).

Muitos governos, seja em nível nacional, estadual ou municipal, ainda estão nos processos iniciais de utilização do e-government (PINA; TORRES; ROYO, 2008; SANDOVAL-ALMAZAN; GIL-GARCIA, 2012; 2010). Muitos não conseguem avaliar em que estágio do processo se encontram. Isso dificulta o planejamento e o desenvolvimento de iniciativas de e-government e o alcance de um nível mais elevando de uso, de forma que melhores serviços possam ser oferecidos, e a democracia possa ser exercida com mais efetividade a partir dessas ferramentas eletrônicas.

Durante os últimos anos, pesquisadores do mundo inteiro têm despendido esforços para auxiliar no desenvolvimento do e-government. Para isso, têm se empenhado na construção de modelos de avaliação. Ainda não existe um consenso de como proceder para avaliar o e-government (GIL-GARCIA; LUNA-REYES, 2007), e, embora exista uma grande variedade de modelos, a maior parte está focada nas avaliações de níveis nacional e estadual. A Organização das Nações Unidas (ONU), por exemplo, tem liderado uma pesquisa internacional em todos os seus países-membros acerca do desenvolvimento do e-government na esfera federal desde 2001 (UNPAN, 2016). Essa pesquisa é realizada a cada dois anos.

A ampla oferta de instrumentos de avaliação nas esferas nacionais e estaduais não supre a carência de instrumentos que contribuam para o aprimoramento da avaliação do e-government na esfera municipal. Além disso, grande parte dos trabalhos realizados traz dados de países considerados mais desenvolvidos (PINA; TORRES; ROYO, 2008; NORRIS; REDDICK, 2013).

$\mathrm{O} e$-government está envolvido por uma complexa rede de relacionamentos que se dá entre tecnologias, organizações, instituições e variáveis de contexto (ROSE; GRANT, 2010; LUNA-REYES; GIL-GARCIA; ROMERO, 2012). Para compreender essa complexidade, modelos de avaliação de e-government deveriam considerar, além dos resultados ou benefícios, aspectos tecnológicos dos sistema e condições em termos organizacionais, institucionais e de variáveis de contexto (LUNA-REYES; GIL-GARCIA; ROMERO, 2012). Existe uma carência de análises que busquem compreender os elementos que potencializam o desenvolvimento do e-government nos distintos territórios e levem em consideração as características específicas de cada região.

Uma das principais vantagens da avaliação do e-government é a possibilidade de melhorar o planejamento por parte do governo (ALMARABEH; ABUALI, 2010). Um melhor entendimento do contexto do e-government proporcionaria subsídios para o planejamento do nível de e-government que se deseja atingir em uma 
determinada esfera de governo. Alguns governos podem optar por atingir a excelência apenas na forma de prestação de serviços, ou, além disso, podem optar por chegar a um nível de excelência também na integração dos cidadãos em processos deliberativos. Isso vai depender da definição de prioridades e do planejamento de cada governo. As diferentes necessidades das comunidades e suas prioridades levam a diferentes modelos de desenvolvimento do e-government (ALMARABEH; ABUALI, 2010).

A participação da sociedade em nível local tem sido vista pela literatura como uma forma de minimizar muitos dos problemas relacionados à ineficácia das políticas públicas, na medida em que essas passariam a ser planejadas mais de acordo com os anseios e necessidades da sociedade (JAEGER; THOMPSON, 2003), além de proporcionar um maior controle social sobre as ações governamentais implementadas em nível local.

Desde o início do século XXI, pesquisadores têm realizado previsões de que o estágio mais avançado do e-government vai transformar substancialmente a forma como os serviços são prestados e a maneira como governos e cidadãos se relacionam. Isso resultará na evolução de um estágio informacional para um efetivamente interativo e deliberativo, no qual os cidadãos estarão no centro dos objetivos e da construção política.

Nesse contexto, este trabalho busca dar conta de dois objetivos principais: (1) identificar o estágio de maturidade do e-government na esfera local; e (2) permitir a compreensão de fatores que impactam em seu desenvolvimento.

Para atender a esses objetivos da pesquisa, a primeira etapa deste trabalho se deteve na análise do grau de maturidade do e-government na esfera municipal. A segunda etapa consistiu na avaliação da relação entre os estágios de maturidade de e-government e variáveis dos contextos político, tecnológico e social.

O trabalho está organizado em cinco seções, incluindo a presente introdução. A segunda seção apresenta breve revisão da literatura sobre e-government, avaliação de e-government, nível de maturidade de e-government e e-government no nível local. A terceira seção descreve os aspectos metodológicos. A quarta seção apresenta os principais resultados deste estudo. Por fim, a quinta seção apresenta alguns comentários finais, sugestões para estudos futuros e limitações deste estudo. 


\section{Revisão de literatura}

\section{O conceito de e-government}

O e-government apresenta um dos conceitos mais interessantes da área de administração pública, foi introduzido na década de 1990 e ainda não está claramente definido e entendido pelos acadêmicos e técnicos da administração pública (MOON, 2002). Tanto é que existe uma variação de termos, como governo eletrônico, governo digital, governo on-line, governo virtual, entre outros, para se referir ao enfoque que é dado ao aumento da sofisticação das tecnologias de informação e comunicação (TICs) e do seu uso pelo governo (GIL-GARCIA; LUNA-REYES, 2006; MOON, 2002).

Da mesma forma como as TICs têm evoluído, o conceito e a definição de e-government evoluíram. O e-government, além de um meio de oferta de informações e serviços via internet, passou a ser visto como uma forma de transformar as relações entre governos, cidadãos e organizações (HOLDEN; NORRIS; FLETCHER, 2003a).

Os conceitos de e-government apresentados pela literatura especializada podem ser agrupados em quatro enfoques mais comuns e, na maioria das vezes, aparecem numa sequência cronológica (GIL-GARCIA; LUNA-REYES, 2006), quais sejam: (1) o uso da TIC; (2) o suporte das ações do governo; (3) o aprimoramento da relação com os cidadãos; e (4) a orientação à agregação de valor aos participantes do processo.

Os conceitos com enfoque maior no uso das TICs foram os primeiros; e seu ponto-chave se limitava ao uso de recursos eletrônicos ou da TIC, ao uso da internet e à acessibilidade de serviços a qualquer hora e de qualquer lugar (NORRIS; FLETCHER; HOLDEN, 2001; WEST, 2004).

Os conceitos com enfoque no suporte às ações do governo tinham como características disponibilização de informações, oferta de serviços totalmente on-line, aprimoramento dos processos, integração entre departamentos e governos (MOYNIHAN, 2004).

Os conceitos com enfoque no aprimoramento das relações com os cidadãos abordam os direitos políticos e o engajamento dos cidadãos, entre outros fatores que dizem respeito à relação governo-cidadão (HO, 2002).

Os conceitos mais atuais buscam, além de considerar as características dos anteriores, encontrar forma de agregar valor ao processo como um todo e a seus participantes. $\mathrm{O}$ desenvolvimento de estratégias para o uso das tecnologias é percebido 
como mais importante do que apenas utilizar as tecnologias com fim em si mesmas (GIL-GARCIA; LUNA-REYES, 2006).

A definição de e-government utilizada neste artigo busca contemplar as complexas características que se consolidaram sobre a temática com o tempo. Desse modo, concebe-se o e-government como:

seleção, implementação e uso de tecnologias de informação e comunicação no governo para fornecer serviços públicos, melhorar a eficácia gerencial e promover valores e mecanismos democráticos, bem como o desenvolvimento de um quadro legal e regulatório que facilite iniciativas intensivas em informação e promova a sociedade do conhecimento (GIL-GARCIA; LUNA-REYES, 2006, p. 639).

\section{O e-government como um fenômeno social complexo}

A tecnologia é vista como uma ferramenta de relações sociais. O contexto social é definido pela integração do conjunto de equipamentos, técnicas, aplicações e pessoas; a infraestrutura que suporta desenvolvimento e uso, as relações sociais e os processos que constroem tal ambiente (KLING; SCACCHI, 1982). É reconhecido que há uma interação dinâmica entre TIC e a estrutura social em que está inserida.

$\mathrm{O}$ desenvolvimento do e-government implica mudanças sociais e organizacionais. Seu sucesso depende de capacidades técnicas, políticas, legais, financeiras, organizacionais, institucionais e de capital humano (LUNA-REYES; GIL-GARCIA; ROMERO, 2012; ZOO; LEE; YOON, 2012). Os aspectos políticos, tecnológicos e sociais compõem uma complexa rede de relacionamento (LUNA-REYES; GIL-GARCIA; ROMERO, 2012) e desempenham um papel estruturante para o desenvolvimento do e-government. Os aspectos tecnológicos dizem respeito à infraestrutura, à capacidade de acesso dos cidadãos e aos mecanismos e às políticas de segurança. Os aspectos políticos dizem respeito à atuação do governo na gestão dos recursos necessários para fornecer os serviços à população. Por sua vez, os aspectos sociais envolvem o ambiente, os resultados e as consequências e possibilidades de participação dos cidadãos.

\section{A avaliação do e-government}

A avaliação do e-government se tornou um processo importante e essencial para a adoção e o desenvolvimento do e-government (LENK; TRAUNMÜLLER, 2002). A partir da avaliação, são possíveis identificar a situação em que o desenvolvimento do e-government se encontra e verificar o desempenho de objetivos,

Teoria e Evidência Econômica - a. 24, n. 50, p. 141-167, jan./jun. 2018 
estratégias e ações realizadas. Além disso, também permite a comparação de diferentes organizações de governo em níveis nacional e internacional (KUNSTELJ; VINTAR, 2004).

Embora existam modelos conceituais, e esforços estejam sendo realizados para avaliar as diferentes dimensões do e-government, ainda faltam metodologias que permitam, de maneira flexível, comparar o fenômeno do e-government de uma forma clara e integral, visto que a maioria dos modelos de avaliação se limita a avaliar apenas os níveis de agregação do e-government (LUNA-REYES; GIL-GARCIA; ROMERO, 2012).

Para se obter um melhor resultado no processo de avaliação do e-government, alguns desafios precisam ser considerados. Entre esses desafios, estão: (1) identificar as várias dimensões envolvidas; (2) identificar e quantificar benefícios; e (3) considerar os contextos social e técnico (ALSHAWI; ALAHMARY; ALALWANY, 2007).

Algumas abordagens de avaliação do e-government buscam identificar o quanto o e-government tem sido utilizado, ou seja, a relação entre serviços disponibilizados versus o uso desses serviços pelos cidadãos (KUNSTELJ; VINTAR, 2004). Outras buscam avaliar o desenvolvimento do e-government a partir de estágios evolutivos (LAYNE; LEE, 2001; UNPAN, 2016; ANTHOPOULOS; REDDICK, 2016; GIL-GARCIA; MARTINEZ-MOYANO, 2007) que vão desde uma presença inicial na internet até o que viria a ser um nível ideal, com totais integração e alinhamento de serviços e participação democrática dos cidadãos (KUNSTELJ; VINTAR, 2004). Também existem iniciativas que buscam integrar essas diferentes visões em uma mesma metodologia de avaliação (LUNA-REYES; GIL-GARCIA; ROMERO, 2012).

\section{A avaliação da maturidade do e-government}

Os pesquisadores que avaliam o e-government a partir de níveis evolutivos buscam identificar em qual estágio o governo se encontra. Nessa perspectiva, são avaliados desde o início do processo ou a adoção do e-government até outras etapas de seu desenvolvimento, rumo à fase final, que seria o auge de uso ou processos totalmente maduros. As avaliações, nesse caso, dependem do número de fases adotadas, visto que existem diferentes proposições por parte de alguns autores.

Um exemplo é o modelo proposto por Layne e Lee (2001), que consideram que o e-government é um fenômeno evolutivo; e, portanto, as iniciativas de e-government acontecem num processo gradual em que as ações e implementações derivam umas das outras. Nesse sentido, o e-government apresenta um modelo de crescimento

Teoria e Evidência Econômica - a. 24, n. 50, p. 141-167, jan./jun. 2018 
que pode ser dividido em quatro estágios: (1) catalogação - os esforços do governo estão voltados para o estabelecimento de uma presença on-line na web e para a diminuição das demandas de informações aos funcionários públicos; (2) transação - as iniciativas do governo estão focadas em conectar seus sistemas internos a interfaces on-line e permitir que os cidadãos interajam com o governo de forma eletrônica; (3) integração vertical - as esferas nacional, estadual e local de governos estão integradas, conectadas para diferentes funções ou serviços públicos; e (4) integração horizontal - os sistemas dão conta da integração entre as diferentes funções e serviços que o governo oferece, de maneira que o cidadão, a partir de um ponto de partida único, consiga ter suas demandas encaminhadas a outros setores ou departamentos governamentais.

Outro exemplo é a avaliação do e-government realizada pela ONU desde 2001, em nível federal, em todos os países-membros, a cada dois anos. O estudo é realizado para gerar o E-Government Development Index (EGDI) ${ }^{1}$ (UNPAN, 2016).

O índice utilizado para mensurar a maturidade dos serviços on-line é o Online Service Index (OSI). Esse índice é baseado em um modelo de quatro estágios, em que cada estágio subsequente depende da existência do anterior para que possa existir, ou seja, a sofisticação de cada um dos estágios leva a um estágio seguinte. Os quatro estágios são: presença emergente; presença melhorada; presença de transações; e presença conectada (UNPAN, 2016). Uma representação de como estão estruturados os estágios é apresentada na Figura 1.

Figura 1 - Os quarto estágios do desenvolvimento dos serviços on-line

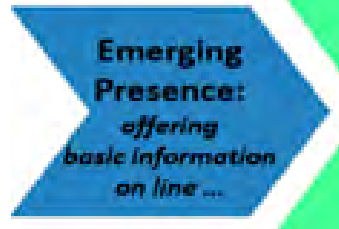

Enhanced

Presence:

greater sources,

e-tools, e-services of

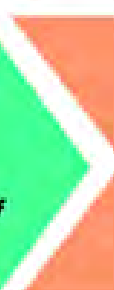

Transactional

Presence:

two ways interactive

opplications, financial

and non financial

transactions ...

Connected

Presence:

WoG, full

interoperability,

G2G, G2C,C2G.

Fonte: <https://publicadministration.un.org/egovkb/en-us/Global-Survey>. Acesso em: 20 ago. 2016.

\section{Presença emergente (emerging presence)}

O primeiro estágio é considerado o mais básico. Sua principal característica é o compartilhamento de informações com os cidadãos. Entretanto, nesse estágio, as informações são consideradas limitadas e básicas. O principal ponto que 
caracteriza esse estágio é a existência de uma página web ou de website, com links para ministérios ou departamentos, ou links para outras esferas de governo podem ou não existir. Algumas informações dos presidentes, assim como mensagens ou documentos, podem estar disponíveis on-line. O restante das informações permanece estático, com mínimas opções aos cidadãos (UNPAN, 2016).

\section{Presença melhorada (enhanced presence)}

O segundo estágio já apresenta algumas características mais aprimoradas do que o primeiro. Nesse estágio, é disponibilizada maior quantidade de informações e documentos sobre políticas públicas e governança, tais como políticas, leis e regulamentos, relatórios e boletins informativos. Essas informações ficam disponíveis e podem até mesmo ser salvas no computador do próprio cidadão. Embora esse estágio seja mais sofisticado, a interação ainda é essencialmente unidirecional, com informações que fluem basicamente do governo para o cidadão (UNPAN, 2016).

\section{Presença transacional (transactional presence)}

O terceiro estágio se caracteriza pela possibilidade de comunicação bidirecional entre governo e cidadãos. Passa a existir a oferta de serviços on-line para os cidadãos como possibilidade de pagar impostos, requisitar documentos de identidade, passaportes, renovação de licenças, entre outras interações entre governo e cidadãos. Além disso, os cidadãos podem, fazendo uso de seu cartão de crédito ou débito ou acessando os canais de seu banco, pagar por serviços públicos relevantes, como violação de veículos automóveis, impostos e taxas de serviços postais. $\mathrm{O}$ fato de as transações acontecerem on-line significa que os serviços ficam disponíveis em tempo integral para os cidadãos (UNPAN, 2016).

\section{Presença conectada (connected presence)}

O quarto e último estágio do modelo representa o nível mais sofisticado nas iniciativas on-line do governo. A principal característica desse estágio está relacionada à integração das interações. Uma outra característica desse estágio é o incentivo do governo para maior participação em processo de tomada de decisão, envolvendo a sociedade em um diálogo mais aberto e participativo. Para isso, o governo 
utiliza, por exemplo, recursos interativos, como o formulário de comentário na web e mecanismos de consulta on-line (UNPAN, 2016).

\section{O e-government no nível local}

O e-government representa mais do que uma revolução na entrega de serviços. Tem potencial para estimular e transformar a filosofia de governos e sua própria organização em todos os níveis (EDMISTON, 2003). Do mesmo modo como pode transformar o governo na esfera nacional, tem condições de fazê-lo na esfera local, movendo governos do paradigma burocrático tradicional para o paradigma do e-government (HO, 2002). Seria a mudança de um paradigma com ênfase em padrões, tendo por base uma departamentalização e focado em operações de baixo custo, para um paradigma que enfatize mais do que as relações internas e suas necessidades de adequações, considerando as relações externas e oportunizando maior colaboração entre governo, cidadãos, organizações e outros envolvidos (HO, 2002).

Entre os principais objetivos de governos locais, estão a modernização dos serviços e a melhora na resposta de demandas cotidianas da população, bem como o suporte para projetos de desenvolvimento econômico local (EDMISTON, 2003). Dessa forma, os serviços públicos na esfera local estão diante do desafio de modernização administrativa, com o intuito de aproximar os serviços dos cidadãos, e, consequentemente, de digitalizar os processos (SÁ; ROCHA; COTA, 2016).

O nível local é onde acontece a maior interatividade com os cidadãos e com os atores locais. É onde ocorre maior oferta de serviços governamentais diretamente voltados ao cidadão (DILLON et al., 2015). O governo local proporciona serviços eletrônicos próprios, fortemente influenciado pelas suas atribuições, pela sua tipologia, pela diferentes distribuições territorial e local, pelos distintos níveis de alfabetismo e idade populacional, pelas diferentes estruturas organizacionais, por uma forte proximidade com o cidadão e com as organizações locais, pelo diferente estado de maturidade das TICs, pelos diferentes canais de disponibilização dos serviços e pelo forte papel influenciador das políticas legislativas local e nacional (SÁ; ROCHA; COTA, 2015). Dessa forma, o ambiente de atuação para os governos locais se mostra desafiador e dinâmico (HATCH; CUNLIFFE, 2006), exigindo agilidade de respostas às condições que se apresentam.

Estudos demonstram que, tanto em países desenvolvidos quanto nos em desenvolvimento, tem havido um esforço significativo para a melhoria das condições na esfera local, que envolvem a desburocratização (KING; COTTERILL, 2007) e a capacidade de resposta, utilizando como estratégia de apoio o e-government para a 
melhora da prestação de serviços aos cidadãos (HO, 2002; MOON, 2002; DILLON et al., 2015). A adoção do e-government, contudo, pode encontrar barreiras, principalmente quando se avança para serviços mais interativos. Entre esses desafios, estão preocupações dos cidadãos com segurança e privacidade. Essas barreiras estão presentes tanto na adoção quanto na evolução do e-government (HOLDEN; NORRIS; FLETCHER, 2003b).

\section{Método}

Esta pesquisa se classifica como um trabalho empírico e quantitativo e tem como principal objetivo identificar o estágio do processo de implementação do e-government na esfera local e as relações entre os estágios de e-government dos municípios com variáveis das dimensões política, tecnológica e social, as quais compõem o conceito de e-government. O Brasil é um país em desenvolvimento no qual os desafios de desenvolvimento e sustentabilidade têm se mostrado cada vez maiores. Das 27 unidades da federação, mais de 70\% estão deficitárias (20 no total ${ }^{2}$, grupo do qual o Rio Grande do Sul, que é composto por 497 municípios, é parte integrante.

Os sujeitos envolvidos na pesquisa são o prefeito municipal e o gestor ou responsável pelo setor de TIC das prefeituras. A escolha desses dois sujeitos se deu porque o primeiro está mais envolvido com as questões estratégicas do município e diretamente ligado às políticas em desenvolvimento; já o segundo está envolvido na providência dos recursos necessários para dar suporte aos projetos de e-government. O contato com os sujeitos da pesquisa aconteceu a partir do apoio da Federação das Associações de Municípios do Rio Grande do Sul (Famurs), entidade representativa da categoria. Os meios para contatar os sujeitos da pesquisa foram e-mail e contato telefônico. Partiu-se do princípio de que todos os 497 municípios do estado do Rio Grande do Sul poderiam participar.

Os dados foram coletados por meio de questionários estruturados enviados via e-mail e, além da comunicação por e-mail, foram realizados dois contatos telefônicos, para solicitar a participação na pesquisa. Participaram os prefeitos e responsáveis pelo setor de TIC de 144 municípios.

Avaliou-se a maturidade do e-government de acordo com os estágios utilizados pela ONU para realizar a pesquisa do EGDI: (1) presença emergente; (2) presença melhorada; (3) presença transacional; e (4) presença conectada (UNPAN, 2016). A atribuição do nível de maturidade do e-government ocorreu de forma sequencial e dependente, ou seja, após atender aos requisitos do nível avaliado e do nível 
anterior, avaliou-se o nível subsequente. Levou-se em consideração, para estabelecer o estágio de maturidade, o atendimento de todas as características dos níveis anteriores.

Nesta pesquisa, considerou-se o desenvolvimento do e-government de forma evolutiva e sequencial. Buscou-se identificar quais municípios estão num processo mais completo do e-government, ou seja, quais estão em um estágio mais avançado atendendo às características de todos os níveis anteriores. A avaliação do estágio do e-government foi realizada dessa forma, para se ter uma visão mais ampla da maturidade do e-government.

As variáveis com as quais se analisou o estágio do e-government foram agrupadas em três dimensões: política, tecnológica e social. As variáveis políticas dizem respeito a variáveis que estão diretamente vinculadas a decisões políticas. As variáveis tecnológicas dizem respeitos a variáveis de suporte tecnológico ao e-government. As variáveis sociais dizem respeito a variáveis de contexto. Nesse caso, os dados vieram de fontes de dados secundários. No Quadro 1, são listadas as variáveis de cada dimensão analisada.

Quadro 1 - Dimensões e variáveis do modelo

\begin{tabular}{|c|c|}
\hline Dimensão & Variável \\
\hline Política & $\begin{array}{l}\text { Apoio dos representantes eleitos } \\
\text { Iniciativas do governo municipal } \\
\text { Retorno dos cidadãos sobre os serviços prestados } \\
\text { Mudança de governo } \\
\text { Participação do Governo Federal }\end{array}$ \\
\hline Tecnológica & $\begin{array}{l}\text { Infraestrutura tecnológica } \\
\text { Treinamento da equipe do setor de TI } \\
\text { Acesso à internet } \\
\text { Disponibilidade de recursos financeiros }\end{array}$ \\
\hline Social & $\begin{array}{l}\text { População* } \\
\text { Î́dice de Desenvolvimento Socioeconômico (Idese) } \\
\text { IDH* } \\
\text { PIB per capita* } \\
\text { PIB município* } \\
\text { Analfabetismo* }\end{array}$ \\
\hline
\end{tabular}

* Variáveis transformadas por Log10 para homogeneização da distribuição nos municípios.

Fonte: elaboração dos autores. 
O software estatístico utilizado foi o SPSS. Para verificar as relações significantes entre as variáveis e os estágios de maturidade de e-government, utilizou-se $o$ teste da variância (ANOVA), com post hoc Tukey; nível de significância de $\mathrm{p} \leq 0,05$.

\section{Resultados}

Nesta seção, constam os principais resultados da pesquisa. Primeiro, mostra-se o estágio de maturidade do e-government, encontrado conforme a metodologia adotada. A seguir, apresentam-se as variáveis com relação de significativa para 0 desenvolvimento do e-government no contexto estudado.

\section{Maturidades do e-government na esfera local}

Os resultados revelaram que o e-government de municípios do estado do Rio Grande do Sul pode ser caracterizado como ainda em estágio inicial. Os dados da pesquisa mostram que ainda existem muitos municípios que permanecem no nível inicial do e-government, e o uso dos recursos de TICs ainda é bastante básico e voltado para necessidades primárias, como suporte e manutenção, e com uma presença na web ainda em fase inicial, orientada para informação.

A pesquisa revelou que $16,7 \%$ dos municípios avaliados estão ainda no estágio I; 73,6\% estão no estágio II; e apenas $9,7 \%$ estão no estágio III.

Tabela 1 - Estágios de maturidade do e-government

\begin{tabular}{l|c|c}
\hline \multicolumn{1}{c|}{ Estágios } & Frequência & Porcentagem \\
\hline Estágio I & 24 & 16,7 \\
Estágio II & 106 & 73,6 \\
Estágio III & 14 & 9,7 \\
Total & 144 & 100,0 \\
\hline
\end{tabular}

Fonte: elaboração dos autores.

Um aspecto que se preocupou em avaliar durante o estudo foi se os resultados obtidos com a investigação poderiam ter alguma influência do tipo de gestão realizada com a estrutura de internet, mais especificamente o website. Para tal, buscou-se identificar se a gestão do site do município era realizada por equipe interna ou terceirizada. Os dados revelaram que 53\% dos sites são administrados e geridos por equipe interna, e que $47 \%$ são administrados e geridos por empresa terceirizada. Pode-se observar, desse modo, que a relação é muito semelhante, próxima a $50 \%$. 
Ao analisar a relação entre a forma de gestão do website e o estágio do e-government, constatou-se que, em nenhum dos estágios, a diferença superou $1 \%$.

Tabela 2 - Estágio de maturidade do e-government por time

\begin{tabular}{l|c|c|c}
\hline \multicolumn{1}{c|}{ Administração do site } & Estágios & Frequência & Porcentagem \\
\hline \multirow{4}{*}{ Equipe interna } & Estágio I & 13 & 17,1 \\
& Estágio II & 56 & 73,7 \\
& Estágio III & 7 & 9,2 \\
& Total & 76 & 100,0 \\
\hline \multirow{3}{*}{ Empresa terceirizada } & Estágio I & 11 & 16,2 \\
& Estágio II & 50 & 73,5 \\
& Estágio III & 7 & 10,3 \\
& Total & 68 & 100,0 \\
\hline
\end{tabular}

Fonte: elaboração dos autores.

A seguir, é apresentado cada um dos quatro estágios de maturidade do e-government, bem como são dispostas as questões que contribuíram para a mensuração e resultados.

\section{Estágio I - Presença emergente}

A principal característica foi o compartilhamento de informações com os cidadãos. O principal requisito desse estágio foi a disponibilização de informações via página web ou website (UNPAN, 2016). Nesse estágio, os principais pontos avaliados foram: a) disponibilização de informações dos gestores públicos no site como mensagens do prefeito, documentos ou legislação; e b) disponibilização de informações sobre os serviços oferecidos pelo poder municipal no site. Sobre informações dos gestores públicos, 95,1\% confirmaram que são disponibilizadas no website, $2,8 \%$ responderam que isso não ocorre, e $2,1 \%$ não souberam responder. No que se refere às informações dos serviços oferecidos pelo poder municipal, 89,6\% confirmaram a disponibilização no site, $8,3 \%$ responderam que não são disponibilizadas, e $2,1 \%$ não souberam responder.

\section{Estágio II - Presença melhorada}

O principal requisito do estágio II foi a disponibilização para consulta ou download de documentos via página web ou website (UNPAN, 2016). Para verificar esse estágio, avaliaram-se: a) a disponibilização de documentos de políticas públicas (saúde, educação, etc.); b) a disponibilização de documentos sobre planejamento municipal; c) a disponibilização de documentos sobre estratégias de e-government; e d) a disponibilização de calendário de reuniões de interesse público, por exemplo. 
Sobre documentos de políticas públicas (saúde, educação, etc.), 68,8\% confirmaram a disponibilização dos documentos, $26,4 \%$ responderam que tais dados não são disponibilizados, e 4,9\% informaram não saber responder. A respeito de documentos sobre planejamento municipal, 61,8\% confirmaram a disponibilização dos documentos, 30,6\% responderam que tais dados não são disponibilizados, e 7,6\% informaram não saber responder. No que se refere a documentos sobre estratégias de e-government, $22,9 \%$ confirmaram a disponibilização dos documentos, $52,8 \%$ responderam que tais dados não são disponibilizados, e 15,3\% informaram não saber responder; além disso, $9 \%$ responderam que não existe estratégia de e-government no município. Sobre calendário de reuniões de interesse público, 22,9\% confirmaram a disponibilização dos documentos, $71,5 \%$ responderam que tais dados não são disponibilizados, e 5,6\% informaram não saber responder.

\section{Estágio III - Presença transacional}

O terceiro estágio se caracterizou pela possibilidade de comunicação bidirecional entre governo e cidadãos. Essa interatividade se deu, na maioria das vezes, a partir de serviços disponibilizados de forma on-line ou recursos específicos que possibilitem ao cidadão contatar o poder público (UNPAN, 2016). Para verificar esse estágio, avaliaram-se: a) a existência de meios para que os cidadãos possam entrar em contato com a administração municipal usando o site; b) a oferta de meios ou serviços on-line, evitando que os cidadãos tenham que se deslocar até a prefeitura para receber atendimento e, em certos casos, possibilitando que realizem pagamentos on-line. Sobre meios para que os cidadãos entrem em contato com a administração municipal, 94,4\% confirmaram a existência desse canal, $4,2 \%$ responderam que ainda não têm essa ferramenta disponível, e 1,4\% informaram não saber responder. Sobre meios ou serviços on-line, como pagamentos, que evitem que o cidadão tenha que se deslocar até a prefeitura para ser atendido, 11,8\% confirmaram a oferta de serviços on-line, $86,8 \%$ responderam ainda não oferecer essa possibilidade, e 1,4\% informaram não saber responder.

\section{Estágio IV - Presença conectada}

A principal característica do nível mais sofisticado nas iniciativas on-line do governo está relacionada à integração das interações. O governo passa a incentivar maior participação dos envolvidos para que se chegar ao nível de participação em processo de tomada de decisão, envolvendo a sociedade em um diálogo mais aberto e participativo (UNPAN, 2016). Para verificar esse estágio, avaliaram-se: a) a existência de meios para que os cidadãos possam expressar sua opinião em assuntos de interesse (por exemplo, painéis de consulta aos cidadãos para elaboração 
de estratégias, consultas eletrônicas, etc.); b) a existência de meios para que os cidadãos possam sugerir aos tomadores de decisão tópicos de interesse da comunidade; c) a existência de programa educacional ou treinamento para participação eletrônica do cidadão. Sobre meios para expressar opinião em assuntos de interesse, $46,5 \%$ confirmaram a existência desse canal, 50,0\% responderam ainda não haver essa possibilidade, e 3,5\% informaram não saber responder. Sobre meios para que os cidadãos possam, de modo proativo, sugerir aos tomadores de decisão tópicos de interesse da comunidade, $42,4 \%$ confirmaram a existência desse canal, $50,7 \%$ responderam ainda não haver essa possibilidade, e 6,9\% informaram não saber responder. Sobre a existência de programa educacional ou treinamento para participação eletrônica do cidadão, 7,6\% confirmaram a existência desse tipo de programa, $85,4 \%$ responderam que ainda não oferecem essa funcionalidade, e 6,9\% informaram não saber responder.

\section{Análise de variáveis de e-government}

A seguir, são apresentados os resultados a partir da análise da relação entre as variáveis de cada uma das dimensões estudadas - política, tecnológica e social - e o nível do e-government.

\section{Dimensão política}

Na dimensão política, foram avaliadas as variáveis: Apoio dos representantes eleitos; Iniciativas do governo municipal; Retorno dos cidadãos sobre os serviços prestados; Mudança de governo; e Participação do Governo Federal. Nessa dimensão, a variável Mudança de governo foi considerada de médio impacto para o e-government, e as demais foram consideradas de alto impacto, mas não demonstraram relação significativa, quando comparadas com os estágios do e-government.

\section{Dimensão tecnológica}

Na dimensão tecnológica, foram avaliadas as variáveis: Infraestrutura tecnológica; Treinamento da equipe do setor de TI; Acesso à internet; e Disponibilidade de recursos financeiros. Nessa dimensão, as variáveis Infraestrutura tecnológica e Disponibilidade de recursos financeiros apresentaram relação significativa com o estágio do e-government. Quanto às demais variáveis, todas foram consideradas de alto impacto para o e-government, mas não demonstraram relação significativa.

O impacto da variável Infraestrutura tecnológica para o desenvolvimento do e-government foi avaliado como: alto para $58,3 \%$; médio para $23 \%$; e baixo para $18,7 \%$. A média foi de 2,31, com um desvio-padrão de 1,286. As variações entre limites inferior e superior da média ficaram em 2,16 e 2,58, respectivamente. 
Gráfico 1 - Média da variável Infraestrutura tecnológica dos municípios estudados por estágios

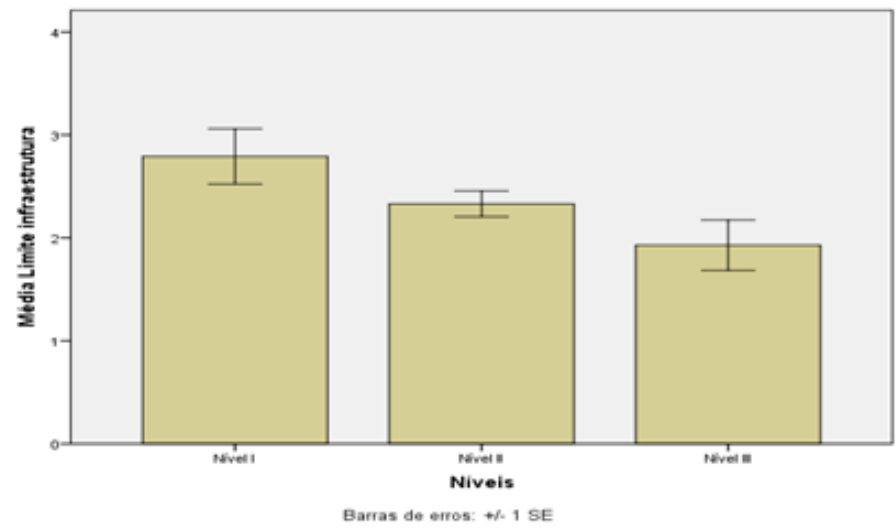

Fonte: elaboração dos autores.

O Gráfico 1 revela que a variável Infraestrutura tecnológica apresenta uma diferença significativa entre os estágios I e III $(p=0,05)$. As médias obtidas para os estágios I, II e III foram 2,79, 2,34 e 1,93, respectivamente. Os desvios-padrão para os estágios I, II e III foram, respectivamente, 1,318, 1,303 e 0,917.

A variável Disponibilidade de recursos financeiros para o desenvolvimento do e-government foi considerada de alto impacto para $54,2 \%$; de médio para $31,3 \%$; e de baixo para $14,6 \%$. A variação entre limites inferior e superior da média ficou em 2,09 e 2,52 , respectivamente.

Gráfico 2 - Média da variável Disponibilidade de recursos financeiros dos municípios estudados por estágios

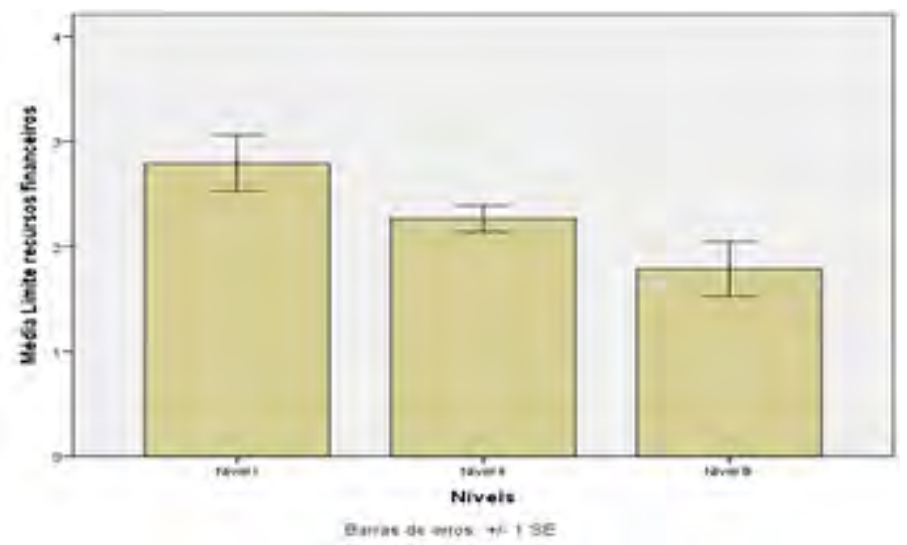

Fonte: elaboração dos autores. 
O Gráfico 2 revela que essa variável apresenta diferença significativa entre os estágios I e III ( $\mathrm{p}=0,05)$. As médias obtidas para os estágios I, II e III foram 2,79, 2,34 e 1,93, respectivamente. Os desvios-padrão para os estágios I, II e III foram, respectivamente, $1,560,1,420$ e 1,292 .

\section{Dimensão social}

Na dimensão social, foram avaliadas as variáveis: População do município; Idese ${ }^{3}$; IDH; PIB per capita; PIB municipal; e Analfabetismo (maiores de 15 anos). Ao analisar a relação das variáveis da dimensão social com os estágios de e-government identificados na esfera local, constatou-se relação significativa apenas nas variáveis População, PIB municipal e Analfabetismo. A variável População teve uma média de 18.234,85 habitantes. As variações entre limites inferior e superior da média ficaram em 12.003,38 e 24.466,31, respectivamente. Os municípios com menor e maior número de habitantes têm, respectivamente 1.421 e 350.824 habitantes. O Gráfico 3 apresenta os resultados da média da população dos municípios avaliados por estágio.

Gráfico 3 - Média da variável População dos municípios estudados por estágios

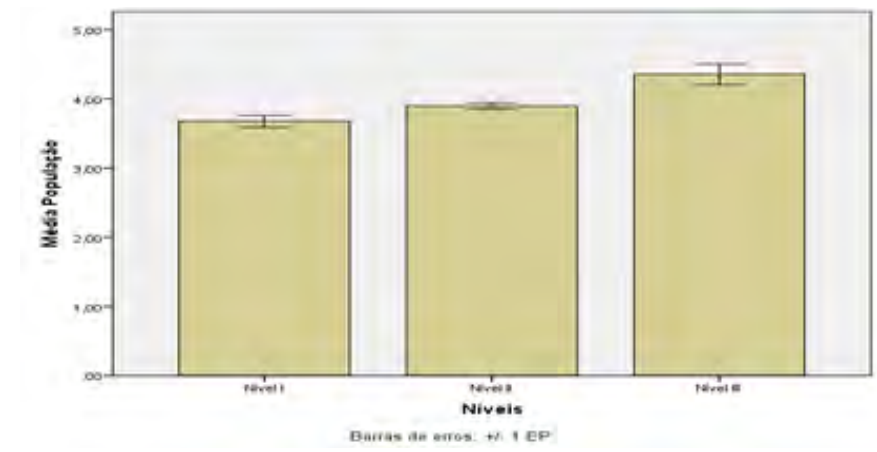

Fonte: elaboração dos autores.

O Gráfico 3 revela que a variável População apresenta uma diferença significativa entre os estágios I e III ( $\mathrm{p}=0,05)$. As médias obtidas para os estágios I, II e III foram 3,67, 3,90 e 4,35, respectivamente. Os desvios-padrão para os estágios I, II e III foram, respectivamente, 0,41, 0,47 e 0,55. 
A variável PIB municipal ${ }^{4}$ teve uma média de $R \$ 582.954,2973$. As variações entre limites inferior e superior da média ficaram em $\mathrm{R} \$ 371.242,7816 \mathrm{e}$ $\mathrm{R} \$ 794.665,8130$, respectivamente. Os municípios com menor e maior PIB municipal têm, respectivamente, $\mathrm{R} \$ 11.777,66$ e $\mathrm{R} \$ 98.696,07$. O desvio-padrão foi de 1.285.247,23857. O Gráfico 4 apresenta os resultados da média do PIB dos municípios avaliados por estágio.

Gráfico 4 - Média da variável PIB municipal dos municípios estudados por estágios

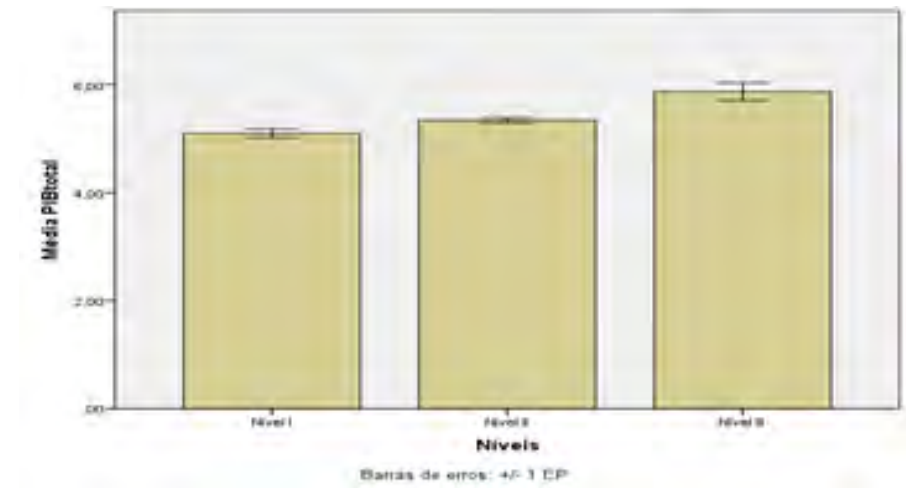

Fonte: elaboração dos autores.

O Gráfico 4 revela que a variável PIB municipal apresenta uma diferença significativa entre os estágios I e III $(p=0,05)$. As médias obtidas para os estágios I, II e III foram 5,1028, 5,3494 e 5,8724, respectivamente. Os desvios-padrão para os estágios I, II e III foram, respectivamente, 0,41617, 0,50781 e 0,61644.

A variável Analfabetismo teve uma média de 6,133262. As variações entre limites inferior e superior da média ficaram em 5,612034 e 6,654491, respectivamente. Os municípios com menor e maior Analfabetismo têm, respectivamente, 0,9461 e 17,3279. O desvio-padrão foi de 3,1642478. O Gráfico 5 apresenta os resultados da média de Analfabetismo dos municípios avaliados por estágio. 
Gráfico 5 - Média da variável Analfabetismo dos municípios estudados por estágios

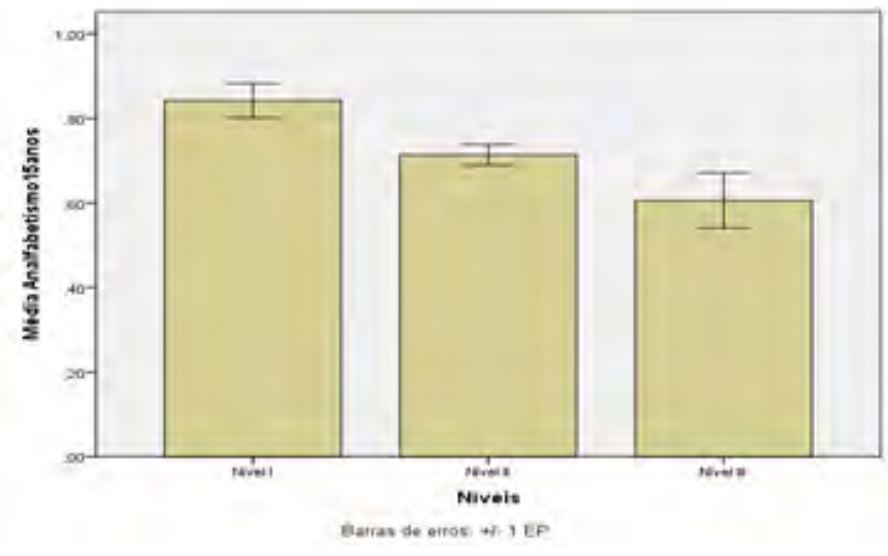

Fonte: elaboração dos autores.

O Gráfico 5 revela que a variável Analfabetismo apresenta uma diferença significativa entre os estágios I e III $(p=0,05)$. As médias obtidas para os estágios I, II e III foram $0,8418,0,7219$ e 0,6058 , respectivamente. Os desvios-padrão para os estágios I, II e III foram, respectivamente, 0,19745, 0,24265 e 0,24457.

\section{Discussão}

O principal objetivo desta pesquisa foi identificar variáveis que possam apresentar relevância para o desenvolvimento do e-government na esfera local. Para melhor compreensão e para fins de orientação do planejamento público municipal, agruparam-se as variáveis nas dimensões política, tecnológica e social. A visão sobre o estágio de desenvolvimento do e-government na esfera local e sua relação com variáveis de contexto podem ajudar os gestores públicos nos processos de tomada de decisão e criação de políticas públicas para o desenvolvimento de suas regiões, levando em consideração os recursos de TIC.

\section{Estágio de maturidade do e-government}

As evidências mostraram que $100 \%$ dos municípios estudados têm página web ou website, superando a média nacional dos municípios, que é de 88\% (CGI, 2016). Entretanto, o uso do e-government ainda está num processo inicial, segundo os quatro estágios de maturidade de e-government (UNPAN, 2016). 
O e-government nos municípios avaliados ainda não evoluiu, como previsto na literatura, para soluções mais transacionais e interativas, que permitam aos usuários acessar serviços remotamente ou participar de forma mais ativa do processo de construção das políticas públicas (COURSEY; NORRIS, 2008).

Os resultados mostram que é maior o percentual de municípios entre os níveis I e II $(90,3 \%), 16,7 \%$ e 73,6\%, respectivamente. Outro ponto que chama atenção é o baixo percentual de municípios que estão no nível III (9.7\%). Isso reflete que a oferta de serviços e a interatividade com os cidadãos são pontos a serem trabalhados na grande maioria dos municípios avaliados.

Os resultados confirmam que os desafios que se apresentam aos governos locais estão cada vez maiores, dado a velocidade com que a sociedade tem se apropriado das novas tecnologias e suas expectativas de encontrar nos órgãos públicos maior oferta de serviços eletrônicos (DE ANDRADE; DE MACÊDO, 2013). Essa oferta ainda é baixa, e os meios de interação com possibilidade de participação deliberativa por parte dos cidadãos, praticamente inexistentes. Isso contradiz o número de cidadãos com acesso à internet no Brasil, que vem aumentando: de 50\% da população em 2014 para 59\% em 2015 (cerca de 51,8 milhões de pessoas) (CGI, 2016).

\section{Variáveis que impactam o e-government}

Identificaram-se cinco variáveis que apresentaram grau de relevância para o desenvolvimento do e-government: as variáveis Infraestrutura de tecnologia e Recursos financeiros na dimensão tecnológica; e as variáveis População, PIB municipal e Analfabetismo na dimensão social.

$\mathrm{Na}$ dimensão tecnológica, a variável Infraestrutura tecnológica apresentou uma relação significativa entre os níveis I e III. Isso reflete a importância da infraestrutura nas fases inicial do e-government e de aumento da oferta de serviços, na qual se depende de recursos tecnológicos mais avançados. No terceiro estágio, por exemplo, para que se possa ofertar serviços e disponibilizar formas de pagamento, faz-se necessária a incorporação de nova tecnologia, como bancos de dados, gateways de conexão com os mecanismos de pagamento, entre outros. Os estágios I e o III precisam de uma infraestrutura de TI preparada, para que os sistemas possam ser iniciados, e as soluções de prestação de serviços possam ser desenhadas e desenvolvidas para o acesso da população.

A variável Disponibilidade de recursos financeiros apresentou uma relação significativa entre os níveis I e III. Isso reflete que, nas fases de maior mudança 
quanto à estrutura e ao suporte tecnológico, os recursos assumem papel determinante para o desenvolvimento do e-government. Para se obter melhoras em infraestrutura e recursos tecnológicos e mais pessoas trabalhando, são necessários mais recursos financeiros, e isso reflete no desenvolvimento do e-government.

Na dimensão social, as variáveis População, PIB municipal e Analfabetismo apresentaram uma relação significativa entre os níveis I e III. Quanto à variável População, confirmou-se o resultado de pesquisa anterior realizada por Moon (2002), de que a população se mostra relacionada ao estágio do e-government. Quanto maior a população, mais propenso se está a um estágio avançado de maturidade do e-government. O que se pode ponderar a partir dessa característica é: por que municípios com maior população têm desenvolvido mais o e-government? Um dos pontos que pode estar diretamente relacionado é a questão dos recursos financeiros. Municípios com maior população tendem a arrecadar mais do que municípios com populações menores. Isso pode ser constatado nesta pesquisa a partir da variável PIB municipal, que se mostrou relacionada com o estágio do e-government, ou seja, quanto maior o montante arrecado pelo município, maior a tendência de um estágio avançado de e-government.

Quanto à variável Analfabetismo, que também apresenta relação com os estágios do e-government, identificou-se que, quanto menor a taxa de analfabetismo no município, maior a tendência de um nível avançado do e-government. Essa constatação reforça o resultado da pesquisa nacional do CGI (2016), em que foi identificado que a utilização dos recursos de e-government é realizada por cidadãos que têm escolaridade mais alta.

Em linhas gerais, observou-se que o estágio do e-government nos municípios estudados ainda se encontra entre as fases I e II, e que as variáveis Infraestrutura tecnológica, Recursos financeiros, População, PIB municipal e Analfabetismo são significativas para o desenvolvimento do e-government e deveriam ser consideradas pelos gestores públicos na elaboração do planejamento público.

\section{Conclusão}

Apresentou-se, neste artigo, um modelo para identificar variáveis com grau de relevância para o desenvolvimento do e-government na esfera local. Agruparam-se essas variáveis em três dimensões: técnica, política e social; como referência, adotou-se o modelo de maturidade utilizado pela ONU para a composição do EGDI.

O modelo se apresentou como mais um recurso para facilitar o planejamento do e-government por gestores municipais em países em desenvolvimento, contribuindo 
para o aprimoramento do planejamento do e-government e fornecendo informações relevantes do contexto local.

O método utilizado foi o método quantitativo, com a participação de municípios do extremo sul do Brasil. Identificou-se que, na maioria dos municípios, o e-government ainda está nos estágios iniciais, entre I e II, e as variáveis Infraestrutura tecnológica, Recursos financeiros, População, Orçamento municipal e Analfabetismo estão relacionadas com o estágio de maturidade do e-government.

Constatou-se que os municípios estudados estão preponderantemente utilizando o e-government como meio de comunicação e informação, não de interação. Existe pouca oferta de serviços on-line em que os cidadãos podam se beneficiar dos recursos de internet para pagamentos ou solicitação de serviços. Oportunidade de participação e colaboração ainda se apresentam como grande desafio a ser superado, e as informações encontradas podem contribuir para o planejamento do e-government.

Reconhece-se que existem algumas limitações quanto ao uso de modelos de maturidade na avaliação do e-government (SANDOVAL-ALMAZÁN; GIL-GARCIA, 2008). Ao mesmo tempo, percebe-se neles uma forma prática de dimensionar o quanto o e-government tem se difundido em dado contexto. Para a avaliação dos níveis, considerou-se que o e-government se desenvolve a partir de uma relação linear, fase após fase, o que poderia não levar em consideração casos em que o e-government se desenvolve em mais de uma fase ao mesmo tempo.

Pesquisas futuras poderiam agregar diferentes variáveis às dimensões política, tecnológica e social, de forma a dar um conjunto maior de informações e auxiliar na elaboração de planejamentos municipais e na tomada de decisão. 


\title{
The development of e-government in the local sphere from the political, technological and social dimensions
}

\begin{abstract}
This paper presents a model to identify variables of relevance for the development of e-government in the local sphere. Its purpose is to contribute to the understanding of the local context from analytical information and to assist the planning of e-government by municipal managers. It also stimulates regional development by helping public servants to understand relevant variables in the local context for solving social problems. The method used was the quantitative method, with the participation of municipalities in the extreme south of Brazil. We have identified that in most municipalities, e-government is still in the early stages, where information technology is basically used as a means of communication. We also identified that the variables technological infrastructure, financial resources, population, municipal budget and illiteracy rate have been shown to be more likely to influence the maturity stage of e-government.
\end{abstract}

Keywords: E-government. Maturity model. Information technology.

\section{El desarrollo del e-government en la esfera local a partir de dimensiones políticas, tecnológicas y sociales}

\begin{abstract}
Resumen
Este estudio presenta un modelo para identificar variables de relevancia para el desarrollo del e-government en el ámbito local. Su objetivo es contribuir a la comprensión del contexto local a partir de la información analítica y ayudar a la planificación del e-government por parte de los administradores municipales. También estimula el desarrollo regional al ayudar a los servidores públicos a comprender variables relevantes en el contexto local para resolver problemas sociales. El método utilizado fue el método cuantitativo, con la participación de los municipios del extremo sur de Brasil. Hemos identificado que en la mayoría de los municipios, el e-government aún se encuentra en las primeras etapas, donde la tecnología de la información se utiliza básicamente como medio de comunicación. También identificamos que las variables infraestructura tecnológica, recursos financieros, población, presupuesto municipal y tasa de analfabetismo han demostrado tener mayor probabilidad de influir en la etapa de madurez del e-government.
\end{abstract}

Palabras clave: E-government. Modelo de madurez. Tecnología de la información. 


\section{Notas}

1 O EGDI é baseado em uma pesquisa que mede a presença on-line de todos os 193 estados-membros da ONU e avalia sites nacionais e como as políticas e estratégias de governo eletrônico são aplicadas em geral e em setores específicos para prestação de serviços essenciais. A avaliação classifica o desempenho do $e$-government dos países em relação uns aos outros, em vez de ser uma medição absoluta. Os resultados são tabulados e combinados com um conjunto de indicadores que incorporam a capacidade de um país para participar na sociedade da informação, sem a qual os esforços de governo eletrônico de desenvolvimento seriam de uso imediato e limitado.

2 Disponível em: <http://especiais.g1.globo.com/economia/2016/raio-x-da-crise-nos-estados/>. Acesso em: 20 fev. 2017.

3 Índice comparativo usado para classificar os municípios do Rio Grande do Sul pelo seu grau de desenvolvimento socioeconômico, publicado anualmente pela Fundação de Economia e Estatística.

4 Unidade de medida $\mathrm{R} \$ 1.000,00$

\section{Referências}

AFRICA, U. N. E. C. F. Framework for a set of e-government core indicators. Partnership On Measuring ICT For Development, n. March, p. 1-54, 2012.

ALFORD, J.; HUGHES, O. Public Value Pragmatism as the Next Phase of Public Management. The American Review of Public Administration, Oaks, CA, v. 38, n. 2, p. 130-148, 2008.

ALMARABEH, T.; ABUALI, A. A general framework for E-Government : definition maturity challenges, opportunities, and success. Europen Journal of Science Research, v. 39, n. 1, p. 29-42, 2010.

ALSHAWI, S.; ALAHMARY, A.; ALALWANY, H. E-Government evaluation factors: citizen's perspect. In: EUROPEAN AND MEDITERRANEAN CONFERENCE ON INFORMATION SYSTEMS. Proceedings... Valencia: Polytechnic University of Valencia, 2007. p. 1-12.

ANTHOPOULOS, L. G.; REDDICK, C. G. Understanding electronic government research and smart city: a framework and empirical evidence. Information Polity, San Antonio, TX, USA, v. 21 , n. 1, p. 99-117, 2016.

CGI. TIC - Governo Eletrônico 2015. [S.l: s.n.], 2016.

COURSEY, D.; NORRIS, D. F. Models of E-Government: Are They Correct? An Empirical Assessment Published by: Wiley on behalf of the American Society for Public Administration Models of E-Government: Are They Correct? New An Empirical Assessment E-Government Perspectives on. Public Administration Review, v. 68, n. 3, p. 523-536, 2008.

DE ANDRADE, Jackeline Amantino; DE MACÊDO, Caroliny Wanderley. Ciência e tecnologia para o desenvolvimento regional. Revista de Políticas Públicas, São Luís, MA, v. 16, n. 1, p. 67-78, 2013.

DILLON, S. et al. A longitudinal study of local E-Government development: the policy maker perspective. In: ECIS, 2015. Proceedings... [S. l.: s. n.], 2015. p. 1-16.

EDMISTON, K. D. State and Local E-Government: prospects and challenges. The American Review of Public Administration, v. 33, n. 1, p. 20-45, 2003. 
GIL-GARCIA, J. R.; LUNA-REYES, L. F. Integrating conceptual approaches to E-Government. In: KHOSROW-POUR, M. (Ed.). Encyclopedia of E-Commerce, E-Government and Mobile Commerce. Hershey: Idea Group Reference, 2006. p. 636-643.

Modelo multi-dimensional de medición del gobierno electrónico para América Latina y el Caribe. Santiago: Naciones Unidas, 2007.

GIL-GARCIA, J. R.; MARTINEZ-MOYANO, I. J. Understanding the evolution of e-government: the influence of systems of rules on public sector dynamics. Government Information Quarterly, v. 24, n. 2 , p. $266-290,2007$.

GRIMSLEY, M.; MEEHAN, A. E-government information systems: evaluation-led design for public value and client trust. European Journal of Information Systems, v. 16, n. 2, p. 134-148, 2007.

HATCH, M. J.; CUNLIFFE, A. L. Organisation theory modern, symbolic, and postmodern perspectives. [S.l: s.n.], 2006.

HEEKS, R. Reinventing Government in the Information Age. [S.l: s.n.], 2002.

HO, A. T.-K. Reinventing local governments and the E-Government initiative. Public administration review, v. 62, n. 4, p. 434-444, 2002.

HOLDEN, S. H.; NORRIS, D. F.; FLETCHER, P. D. Electronic government at the grass roots: contemporary evidence and future trends. In: HAWAII INTERNATIONAL CONFERENCE ON SYSTEM SCIENCES, 36., 2003, Hawaii. Proceedings... Hawaii, 2003a. v. 5, p. 134c (1-8).

Electronic government at the local level: progress to date and future issues. Public Performance \& Management Review, v. 26, p. 325-344, Apr. 2003b.

HOOD, C. The "new public management" in the 1980s: variations on a theme. Accounting, organizations and society, v. 20, n. 2-3, p. 93-109, 1995.

IRANI, Z.; LOVE, P. E. D. Evaluating Information Systems: public and private sector. [S.l: s.n.], 2008.

JAEGER, P. T.; THOMPSON, K. M. E-government around the world: lessons, challenges, and future directions. Government information quarterly, v. 20, n. 4, p. 389-394, 2003.

KING, S.; COTTERILL, S. Transformational government? The role of information technology in delivering citizen-centric local public services. Local Government Studies, v. 33, n. 3, p. 333-354, 2007.

KLING, R.; SCACCHI, W. The web of computing: computer technology as social organization. [S.l: s.n.], 1982. v. 21.

KUNSTELJ, M.; VINTAR, M. Evaluating the progress of e-government development: a critical analysis. Information Polity: the International Journal of Government \& Democracy in the Information Age, v. 9, n. 3-4, p. 131-148, 2004.

LAYNE, K.; LEE, J. 1.2 Developing a fullly functional e-government: a four stage model. Government Information Quaterly, v. 18, p. 122-136, 2001.

LENK, K.; TRAUNMÜLLER, R. Electronic government: where are we heading? In: EGOV, 1., 2002, Aix-en-Provence, France. Proceedings... Aix-en-Provence, Sept. 2002. v. 2456, p. 1-9. 
LUNA-REYES, L. F.; GIL-GARCIA, J. R.; ROMERO, G. Towards a multidimensional model for evaluating electronic government: proposing a more comprehensive and integrative perspective. Government Information Quarterly, v. 29, n. 3, p. 324-334, 2012.

MOON, M. J. The evolution of E-Government among municipalities: rhetoric or reality? Public Administration Review, v. 62, n. 4, p. 424-433, 2002.

MOYNIHAN, D. P. Building secure elections: e-voting, security, and systems theory. Public administration review, v. 64, n. 5, p. 515-528, 2004.

NORRIS, D. F.; FLETCHER, P. D.; HOLDEN, S. H. Is your local government plugged in? Highlights of the 2000 electronic government survey. Washington, DC: International City/ County Management Association, 2001. p. 1-12.

NORRIS, D. F.; REDDICK, C. G. 1.0-Local E-Government in the United States: transformation or incremental change? Public Administration Review, v. 73, p. 165-175, 2013.

PINA, V.; TORRES, L.; ROYO, S. E-government evolution in EU local governments: a comparative perspective. In: EUROPEAN CONFERENCE ON E-GOVERNMENT, 2008. Proceedings... [S. l.]: ECEG, 2008. p. 445-454.

ROSE, W. R.; GRANT, G. G. Critical issues apertaining to the planning and implementation of E-Government initiatives. Government Information Quarterly, v. 27, n. 1, p. 26-33, 2010.

SÁ, F.; ROCHA, Á.; COTA, M. P. Potential dimensions for a local e-Government services quality model. Telematics and Informatics, v. 33, n. 2, p. 270-276, 2016.

Preliminary dimensions for a quality model of Electronic Local Government sevices. In: Information Systems and Technologies (CISTI), 2015, 10., Iberian Conference on. IEEE, 2015. p. 1-10.

SANDOVAL-ALMAZAN, R.; GIL-GARCIA, J. R. Assessing local E-government: an initial exploration of the case of Mexico. In: INTERNATIONAL CONFERENCE ON THEORY AND PRACTICE OF ELECTRONIC GOVERNANCE, 4., 2010, New York. Proceedings... New York: ICEGOV, May 2010. p. 61-65.

Are government internet portals evolving towards more interaction, participation, and collaboration? Revisiting the rhetoric of e-government among municipalities. Government Information Quarterly, v. 29, n. suppl. 1, p. S72-S81, 2012.

Limitations of evolutionary approaches to E-Government. In: GARSON, G. D.; KHOSROW-POUR, M. Handbook of Research on Public Information Technology. Hershey: IGI Global, 2008. p. 22-30.

SANTOS, M. A revolução tecnológica e o território : realidades e perspectivas. Terra livre, v. 9, p. 7-17, 2015.

UNPAN. United Nations E-Government Survey 2016: E-Government in Support of Sustainable Development. New York: United Nation, 2016.

WEST, D. M. E-Government and the transformation of service delivery and citizen attitudes. Public Administration Review, v. 64, n. 1, p. 15-27, 2004.

ZOO, H.; LEE, H.; YOON, J. Assessing the E-Government Maturity for Public Sector Innovation in Developing Countries: Case of National Informatization Assessment Tool (NIAT). In: INTERNATIONAL CONFERENCE ON SOCIAL IMPLICATIONS OF COMPUTERS IN DEVELOPING COUNTRIES, 14., Yogyakarta, Indonesia, 2017. Proceedings... New York: LLC, 2017. p. 778-789. 